\title{
Influencia del uso de redes sociales en la venta de productos: Microempresa Color Rosa
}

\author{
Fecha de recepción: 01-04-2021 • Fecha de aceptación: 28-05-2021 • Fecha de publicación: 10-06-2021
}

\author{
Dayana Lizbeth Campoverde Ochoa \\ Asiacar, Ecuador \\ dayana_campoverde92@hotmail.com \\ https://orcid.org/0000-0002-9367-1182
}

\section{RESUMEN}

En la actualidad, las redes sociales son un aliado estratégico de los entes económicos para generar ventas, además de ser las estrategias de marketing de gran importancia para potenciar la publicidad y elevar los márgenes de ganancia de medianas y grandes empresas. De esta manera, la presente investigación que se planteó responde a lo anterior, teniendo como objetivo analizar la influencia del uso de redes sociales en la venta de productos de la Microempresa Color Rosa de Ecuador. El estudio es empírico - descriptivo, a su vez, se aplicó una encuesta a posibles clientes que se encuentran situados en el sector de Calderón, ubicación actual de la microempresa, adicional, se obtuvo datos estadísticos de la página de Facebook de Color Rosa, donde se presenta el alcance de sus publicaciones. El hallazgo más importante denota que la mayoría de encuestados usan la plataforma de Facebook para buscar y comprar productos o servicios de su interés, y la información que se presenta en esta red influye en la decisión de compra. 


\section{ABSTRACT}

Currently, social networks are a strategic ally of economic entities to generate sales, in addition to being marketing strategies very important to enhance advertising and increase profit margins of medium and large companies. Thus, the present research responds to the above, with the objective of analyzing the influence of the use of social networks in the sale of products of the Microcompany Color Rosa of Ecuador. The study is empirical - descriptive, in turn, a survey was applied to potential customers who are located in the sector of Calderon, current location of the microenterprise, in addition, statistical data was obtained from the Facebook page of Color Rosa, where the scope of their publications is presented. The most important finding shows that most respondents use the Facebook platform to search for and purchase products or services of interest to them, and the information presented on this network influences the purchase decision.

KEYWORDS: social networks, digital marketing, strategies, sales. 


\section{Introducción}

Las redes sociales son aplicaciones que la sociedad usa para subir contenidos de su vida e información en general. Actualmente, la mayoría de personas, por no decir casi todas, tienen acceso a estas redes, y su uso para crear páginas de emprendimientos y negocios ha aumentado relativamente, debido a lo fácil y llamativo que es llegar a la mente del consumidor aplicando ciertas claves de marketing digital. Según Mejía (2018); consultor y speaker de marketing digital, las redes sociales son una comunidad de personas que se conectan a una plataforma de Internet que tienen intereses en común. Es decir, son aplicaciones que permiten al usuario interactuar con otros usuarios.

Una de las redes sociales más utilizadas en todo el mundo es Facebook, en esta las personas puedan interactuar y publicar desde fotos, videos hasta los productos y servicios que ofrecen. El reconocido especialista Mejía (2020) menciona en su blog que es una red social que conecta a familiares y amigos o personas con los mismos intereses y es por esta razón que las empresas usan este entorno, por la frecuencia de uso de esta red, de manera que el posible cliente puede evidenciar la veracidad del negocio e incluso puede publicar su opinión con respecto a su experiencia de compra, permitiendo que, con el uso de estas herramientas, el emprendedor o empresario pueda aumentar su embudo de ventas.

Otra herramienta es WhatsApp Business, una aplicación que las empresas utilizan a menudo para tener una comunicación más rápida, directa y efectiva con los clientes, mediante mensajes automatizados (Mejía, 2020).

Comparando con la investigación titulada "Impacto del uso de las redes sociales como estrategia de marketing en las ventas de sociedad predial y mercantil Milatex S.A." se concluye que el uso de las redes sociales, como canal de publicidad y sus ventajas, es un tema de interés global. $Y$ cada vez son más las empresas que se unen a esta era digital, siendo la aplicación de Facebook la más utilizada por estas; para aprovechar las cualidades en cuanto a cantidad de información, segmentación, capacidad de control y el precio que ofrecen las redes sociales, a diferencia de los demás canales de publicidad (Cabrera, 2018).

En la investigación realizada en el año 2015 sobre estrategias de marketing a través del uso de redes sociales, se determinó mediante tabulaciones que estas plataformas son los canales actuales para difundir información y para que el cliente encuentre los productos o servicios de su interés, ya que existen varias empresas o personas que ponen a disposición sus artículos, y que cada uno se diferencia por las estrategias que implementan (Torres \& Yupangui, 2015).

Así mismo se toma como referencia otro trabajo relacionado al tema presentando, donde en función de los métodos que aplicaron concluyen que la red social más utilizada es Facebook con $82,94 \%$, debido a que es fácil y rápido contactarse con familiares y amigos, y este medio ofrece mucho entretenimiento a los usuarios (Tica, Isabel, Ruiz y Augusto, 2018).

A su vez, en el trabajo de investigación sobre un manual de uso de las redes sociales en la promoción de MiPymes de Guayaquil, el autor concluye que las redes sociales han tomado poder 
en los últimos tiempos, sobre todo para la comercialización, y mencionan que si las MIPYMES manejan adecuadamente estos medios, los negocios pueden crecer, aumentar sus beneficios económicos e incrementar las fuentes de trabajo. El crecimiento de seguidores en las redes sociales viene de la mano con la rentabilidad, confianza y reputación de la empresa o negocio (Fuentes, 2018).

En base a lo descrito, el objetivo de la presente investigación es analizar la influencia del uso de redes sociales en la venta de productos de la Microempresa Color Rosa que inició sus actividades en el año 2015, en la ciudad de Quito, idea de la emprendedora Daniela Redrobán que ofrece una gama de arreglos con frutas, globos, flores, entre otros.

\section{Metodología}

La investigación es empírica pues se basa en la experiencia (Rodríguez \& Pérez, 2017). El tipo de investigación es descriptiva, acorde con la realidad de los acontecimientos (Cabezas, Andrade \& Torres, 2018). La técnica de investigación aplicada es la encuesta, ya que permite levantar datos referentes al uso de las redes sociales, misma que constó de 14 preguntas cerradas y de opción múltiple revisadas y aprobadas por el Mg. Sebastián Pérez, profesor universitario de la Universidad Tecnológica Israel.

Para determinar el tamaño de la muestra se aplicó la técnica de muestreo aleatorio simple sobre la población de Calderón, dando como resultado 384 personas a ser encuestadas.

Conjuntamente se obtuvo datos estadísticos de la página de Facebook de Color Rosa, para conocer el alcance que han logrado sus publicaciones y se utilizó el método cuantitativo en la medición de las características de los fenómenos sociales que permite al investigador obtener datos y luego realizar un análisis de estos (Reyes, 2016).

\section{Resultados}

Partiendo de los resultados más relevantes de la encuesta aplicada en esta investigación, se puede mencionar que el $84.1 \%$ de personas encuestadas manifestó que es Facebook la red social de mayor frecuencia de uso, seguido de Instagram en un $42.7 \%$, Tik Tok con un $15.7 \%$, Twitter con un $15.4 \%$, YouTube con un $47 \%$, WhatsApp con un total de $6 \%$, tal como se evidencia en la Figura 1. 


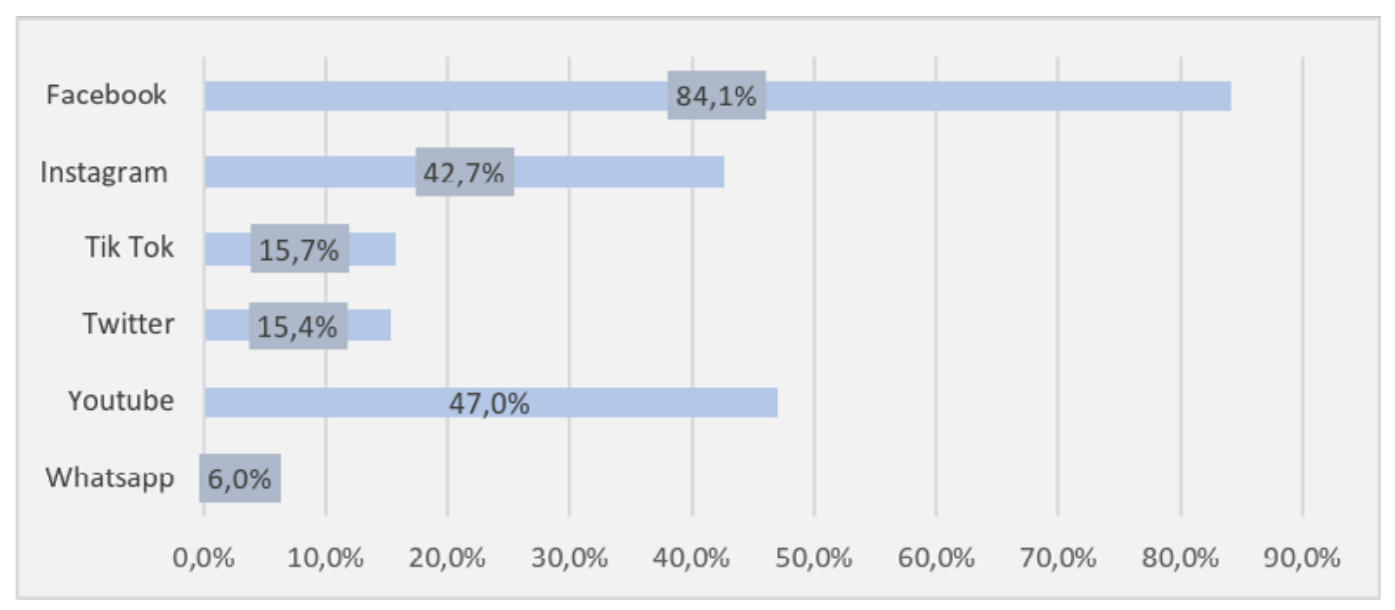

Figura 1. Análisis de las redes sociales más utilizadas en Calderón.

Fuente: elaboración propia

Por otra parte, como se observa en la Tabla 1, las respuestas indicaron que Facebook, con un $80.6 \%$, es la red social en donde según este estudio los usuarios observan mayor cantidad de publicidad, debido a que la mayoría de empresas y emprendedores están utilizando esta herramienta para tener mayor presencia en el mundo del Internet, YouTube es la plataforma siguiente con un $39,5 \%$, Instagram posee el $25 \%$, y las siguientes redes sociales obtuvieron porcentajes bajos con respecto a la publicidad que emiten.

Tabla 1.

Red social que contiene mayor publicidad

\begin{tabular}{|l|l|}
\hline \multicolumn{1}{|c|}{ Red Social } & \multicolumn{1}{c|}{ Respuestas } \\
\hline Facebook & $80,6 \%$ \\
\hline YouTube & $39,5 \%$ \\
\hline Instagram & $25,0 \%$ \\
\hline Tik Tok & $5,4 \%$ \\
\hline Twitter & $2,4 \%$ \\
\hline
\end{tabular}

Fuente: elaboración propia

De igual forma se identificó que el 92,8\% de encuestados (Figura 2) opinan que las redes sociales si son una buena plataforma para ofertar un abanico de opciones para escoger los productos o servicios, afirmando que las redes sociales son la mejor manera de llegar a las personas en la actualidad, ya que la gran mayoría de personas tienen actualmente acceso a redes sociales. 


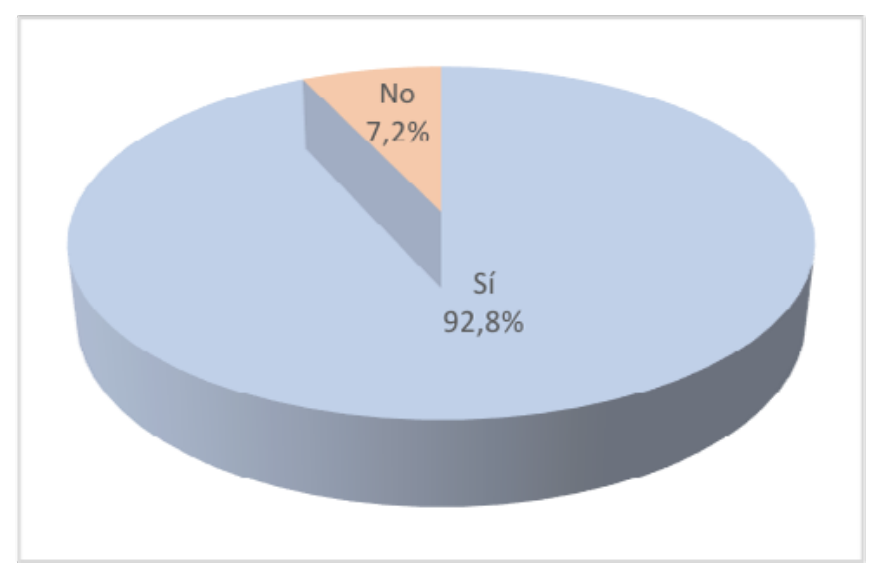

Figura 2. Estudio referente a si las redes sociales son útiles para ofertar productos y servicios

Fuente: elaboración propia

Analizando los datos obtenidos (Figura 3), de la muestra encuestada, el $77.9 \%$ opina que el contenido y publicidad en redes sociales pertenecientes a empresas o emprendimientos sí influyen en su decisión de compra, siendo éste un dato importante para la presente investigación ya que mediante la aplicación de marketing digital las publicaciones tienen mayor impacto en los compradores.

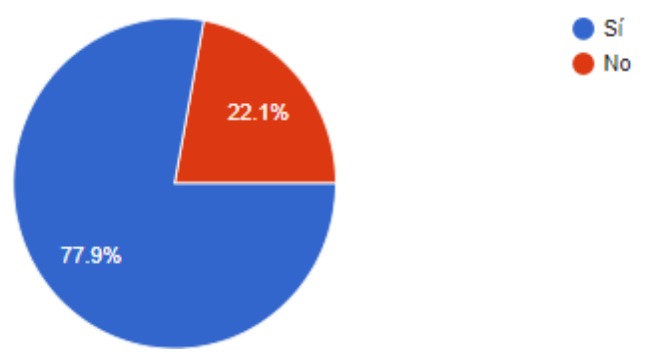

Figura 3. Influencia del contenido y publicidad de las redes sociales en la decisión de compra

Fuente: elaboración propia

Adicional, se puede indicar que el $84,2 \%$ de encuestados (Tabla 2) determinó que Facebook es la plataforma que más usan las personas para buscar productos o servicios de interés, entonces la aplicación del marketing digital en esta plataforma permite crear contenido de calidad que atrae a clientes potenciales, Instagram es la segunda red con mayor uso con el $25.6 \%$, YouTube con $10,9 \%$ y las redes sociales de menor uso son Tik Tok y Twitter y WhatsApp. 
Tabla 2.

Red social más utilizada para conocer y comprar productos o servicios

\begin{tabular}{|l|l|}
\hline \multicolumn{1}{|c|}{ Red Social } & \multicolumn{1}{c|}{ Respuestas } \\
\hline Facebook & $84,2 \%$ \\
\hline Instagram & $25,6 \%$ \\
\hline YouTube & $10,9 \%$ \\
\hline Tik Tok & $4,4 \%$ \\
\hline Twitter & $2,5 \%$ \\
\hline WhatsApp & $0,12 \%$ \\
\hline
\end{tabular}

Fuente: elaboración propia

Otros resultados que se destacan en el presente proyecto son que de 384 encuestas aplicadas en total, el rango predominante de edad de los encuestados esta entre 26 años a 30 años, 367 personas que representan el $98.9 \%$ indican que usan o usaron en algún momento redes sociales siendo la de mayor frecuencia de uso Facebook con un 84.1\%, seguido de Instagram en un $42.7 \%$, Tik Tok con un 15.7\%, Twitter con un 15.4\%, YouTube con un 47\%, WhatsApp con un $6 \%$, entonces, al ser Facebook la red social con mayor frecuencia de uso, se consultó a los encuestados el porqué de su uso y algunos de sus comentarios son que se trata de una red muy versátil en contenido, entretenida, dinámica y muy amigable con los usuarios.

En lo que respecta al tiempo de interacción al día con las redes sociales, el 34.9\% de los encuestados interactúan de 1 a 2 horas diarias en redes sociales, el 24.5\% interactúa de 15 minutos a una hora diaria, el $30.4 \%$ de 3 a 4 horas siendo estos los rangos más representativos en lo que se refiere a la interacción en redes, el rango predominante de 1 a 2 horas arroja que es un tiempo prudente para observar publicidad de varias empresas, siendo justamente en Facebook con un total de $80.6 \%$, en donde según este estudio los usuarios observan mayor cantidad de publicidad.

Igualmente se identificó que el $92.8 \%$ de encuestados opinan que las redes sociales si son una buena plataforma para ofertar productos o servicios, al mismo tiempo, consideran que las redes sociales son la mejor manera de llegar a las personas en la actualidad debido a que la gran mayoría de personas tienen actualmente acceso a redes sociales. El 80.2\% de personas encuestadas indican que han realizado una compra en alguna red social, y el $56.6 \%$ mencionan que sus compras por este medio han sido de forma satisfactoria.

Analizando los datos obtenidos, de la muestra encuestada el $77.9 \%$ opina que el contenido y publicidad en redes sociales pertenecientes a empresas o emprendimientos sí influyen en su decisión de compra, siendo éste un dato importante para la presente investigación por que se concluye que el uso de redes sociales influye de manera positiva en las ventas y que es Facebook, con un total de $84,2 \%$, la plataforma que más usan las personas para buscar productos o servicios de interés.

Los datos obtenidos de la encuesta también arrojaron que la mayoría de participantes regala 
arreglos florales o frutales en ocasiones como cumpleaños y los principales factores que influyen en la compra de productos o servicios son el precio en su mayoría con el $73.6 \%$, seguido de la calidad con el $67.7 \%$, la atención al cliente con un total de $38.8 \%$, las opiniones de otros compradores con un total de $28 \%$ y las publicaciones de los modelos con el $27 \%$. Finalmente, el 92.5\% de encuestados dice no conocer a la empresa Color Rosa y el $7.5 \%$ que, si conoce a la empresa, indican que ha sido por medio de Facebook.

Adicionalmente, se obtuvo las estadísticas de Facebook de la empresa Color Rosa correspondientes al período del 21 de diciembre de 2020 al 17 de enero 2021, con la observación directa de estos datos se ha considerado el análisis de: "alcance de las publicaciones", "interacción de las publicaciones" los nuevos "me gusta" de la página, los registros de "nuevas visitas" a la página, los "nuevos seguidores" como principales impulsadores de la influencia que llega a tener esta red social para beneficio de la empresa Color Rosa y como enfoque a la problemática planteada en este trabajo, a continuación los resultados en detalle:

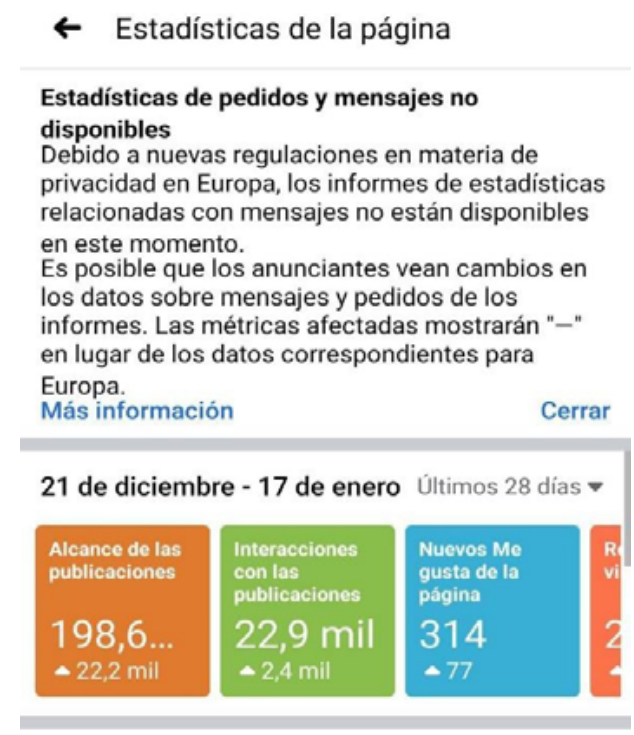

Figura 4. Estadísticas de la página de Facebook de Color Rosa

Fuente: página de Facebook de Color Rosa

En cuanto al alcance de las publicaciones, durante el período de diciembre 21 de 2020 a enero 17 de 2021 la página de Color Rosa obtuvo 198.600 de alcance total (Figura 4), esto se refiere a la cantidad total de personas a las que la empresa Color Rosa llegó con sus publicaciones y que a su vez interactuaron en ella, esto en comparación al periodo referencial de noviembre de 2020 de la misma empresa nos refleja que existe un incremento de $22.2 \%$ de personas nuevas que interactúan con la página.

Para la interacción de las publicaciones, durante el período de diciembre 21 de 2020 a enero 17 de 2021 la página de Color Rosa obtuvo 22.900 interacciones en su página, esto se refiere a la cantidad de: me gusta, comentarios, las veces que se compartió una publicación, clics de las publicaciones, de la misma manera, en comparación al período correspondiente a noviembre de 2020, existe un incremento del $2.4 \%$ de nuevas interacciones en la página de Color Rosa. Mientras 
que para los nuevos me gusta, como parte de la interacción de las publicaciones a la página de Color Rosa durante el período de diciembre 21 de 2020 a enero 17 de 2021 la página obtuvo 314 me gusta (Figura 5).

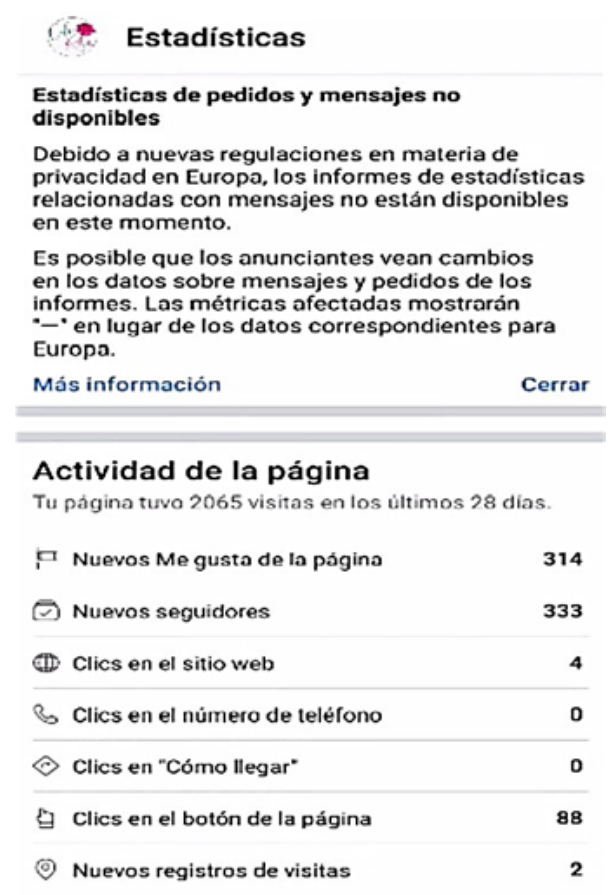

Figura 5. Actividad de la página de Facebook de Color Rosa

Fuente: página de Facebook de Color Rosa

A su vez, refiriéndose a los nuevos seguidores, durante el período de diciembre 21 de 2020 a enero 17 de 2021 la página obtuvo 333 nuevos seguidores a su página de Facebook de acuerdo a la figura anterior. 


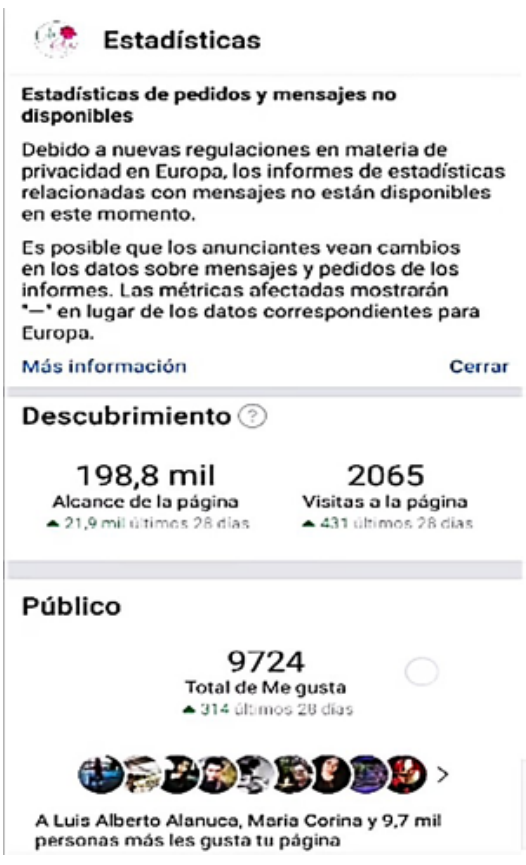

Figura 6. Visitas de la página de Facebook de Color Rosa

Fuente: página de Facebook de Color Rosa

Como se evidenció en la Figura 6, en cuanto a las nuevas visitas a la página, la actividad de la página correspondiente al período de diciembre 21 de 2020 a enero 17 de 2021 fue de un total de 2065 visitas nuevas.

Comparando los resultados obtenidos con un estudio realizado en el año 2018 acerca del impacto que tienen las redes sociales en las ventas de Food Truck, se puede notar que existe similitud en que, cada vez se maneja más las redes sociales predominando el uso de Facebook tanto por empresas grandes, como pequeños negocios, debido a que es un medio por el cual se dan a conocer y pueden captar más clientes e incrementar sus ventas.

En base a las preguntas de las encuestas aplicadas en el trabajo investigativo de Valdiviezo, se observa que, de un total de 232 personas encuestadas, el $96.1 \%$ utilizan redes sociales para promocionar su negocio y el $61,6 \%$, es decir, 143 encuestados indicaron que es Facebook el medio donde lo hacen debido a que gran parte de la población se encuentra en esta red social.

Otro dato interesante que obtuvo la autora es que 171 personas, es decir el $73.7 \%$, creen que estar presentes en las redes sociales si influye para que el cliente realice una compra, debido a que el contenido publicado atrae al consumo por parte de los clientes, además, Valdiviezo, basándose en un estudio realizado por social media marketing el 13 de febrero de 2014, indica que "el $74 \%$ de los consumidores busca información en las redes sociales para poder decisiones de compras y de este modo hacer que influya en las ventas de los productos y servicios de diferentes marcas" (Valdiviezo, 2018).

Sin embargo, así como las redes sociales pueden ser de beneficio, también existen ciertas 
desventajas de su uso, como se indica en el trabajo de investigación realizado en el año 2019, donde se menciona que la publicidad puede ser engañosa y generar ventas a base de una farsa, es decir, estafas por las redes sociales que vulneran los derechos de los clientes al no cumplir con la calidad o expectativa que se genera por un anuncio y como consecuencia se crea el desprestigio y la desconfianza para realizar compras por estos medios.

Las redes sociales sirven como medio publicitario debido a que tienen mayor alcance, no obstante, hay que tener en cuenta que no todo lo que se publica es real, y para no verse afectado por estas circunstancias se puede recabar información con la opinión y experiencia de otros clientes. Es necesario que los post en las redes sociales sean reales para que este canal de comunicación sirva de ayuda para generar ventas (Jiménez \& Flores, 2019).

\section{Conclusiones}

Son varios los beneficios que se obtiene cuando una empresa o microempresa utiliza las redes sociales para generar publicidad, pues en la actualidad, éstas, son el primer contacto con el cliente.

Facebook se posiciona como la red social más utilizada por los usuarios y negocios debido a la fácil interacción y manejo de esta plataforma, permitiendo ofrecer y encontrar variedad de servicios y productos al gusto de cada consumidor.

Mediante la aplicación de estrategias de marketing digital se puede captar una mayor cantidad de clientes generando un incremento de ventas y una posición en el mercado.

Existen varias herramientas gratuitas y de pago que se pueden aplicar para potenciar el contenido en las redes sociales, permitiendo ser innovadores, eficientes, únicos y cumplir con los objetivos y metas establecidas. 


\section{Referencias}

Cabezas, E., Andrade, D. \& Torres, J. (2018). Introducción a la metodología de la investigación científica. (1 ra ed.). Recuperado de http://repositorio.espe.edu.ec/jspui/bitstream/21000/15424/1//ntroduccion\%20a\%20 la\%20Metodologia\%20de\%20la\%20investigacion\%20cientifica.pdf

Cabrera, M. (2018). Impacto del uso de las redes sociales como estrategia de marketing en las ventas de sociedad predial y mercantil Milatex S.A. (Tesis de maestría). Universidad Católica de Santiago de Guayaquil, Ecuador.

Fuentes, M. (2018). Manual de uso de las redes sociales en la promoción de MiPymes de la Av. Víctor Emilio Estrada entre Ficus y Miguel Aspiazu de la ciudad de Guayaquil, 2018. (Tesis de pregrado). Universidad de Guayaquil, Ecuador.

Jiménez, S. \& Flores, J. (2019). Análisis de la publicidad engañosa en redes sociales Facebook e Instagram de establecimientos de comida rápida del norte de la ciudad de Guayaquil. Revista Observatorio de la Economía Latinoamericana. Recuperado de https://www.eumed.net/rev/oel/2019/03/publicidad-enganosa-redes.html

Mejía, J. (17 de agosto 2018). Qué son las redes sociales: beneficios y cuáles son las más usadas. [Entrada de blog]. https://www.juancmejia.com/redes-sociales/que-son-las-redes-sociales-beneficios-y-cuales-sonlas-mas-utilizadas/

Mejía, J. (2 de abril de 2020). Diferencias entre facebook, instagram y twitter ¿Qué tipo de contenido es mejor para cada uno? [Entrada de blog]. https://www.juancmejia.com/y-bloggers-invitados/facebook-twitter-e-instagram-que-tipo-de-contenido-es-mejor-para-cada-uno/

Mejía, J. (11 de mayo 2020). WhatsApp para empresas (WhatsApp business): Qué es, beneficios y funcionalidades + Video. [Entrada de blog]. https://www.juancmejia.com/marketing-digital/whatsapp-para-empresas-whatsapp-business-que-es-beneficios-funcionalidades-y-usos-en-los-negocios/

Reyes, M., (2016). Metodología de la investigación. Sexto Semestre. México.

Rodríguez, A. \& Pérez, A. (2017). Métodos científicos de indagación y de construcción del conocimiento. Revista Escuela de Administración de Negocios, núm. 82, pp. 1-26. Doi: https://doi.org/10.21158/01208160. n82.2017.1647

Tica, E., Isabel, G., Ruiz, P., \& Augusto, D. (2018). El marketing digital en las redes sociales Facebook, Linkedln y YouTube y su influencia en la fidelización de los clientes de la empresa Atanasovski corredores de seguros. (Tesis de pregrado). Universidad Peruana de Ciencias Aplicadas, Lima, Perú. http://hdl.handle. net/10757/623548 
Torres, C., \& Yupangui, M. (2015). Proyecto de aplicación de estrategias de marketing viral a través del uso de redes sociales para promocionar los productos del mercado artesanal del distrito metropolitano de Quito. ( Tesis de pregrado). Universidad Politécnica Salesiana Sede Quito, Ecuador.

Valdiviezo, G., (2018). Estudio comparativo del impacto de las redes sociales en las ventas de food truck en el distrito metropolitano de Quito y los Valles. (Tesis de pregrado). Universidad de las Fuerzas Armadas, Quito, Ecuador. 


\section{Copyright (c) 2021 Dayana Lizbeth Campoverde Ochoa}

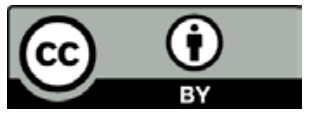

Este texto está protegido bajo una licencia internacional Creative Commons 4.0.

Usted es libre para Compartir - copiar y redistribuir el material en cualquier medio o formato - y Adaptar

el documento - remezclar, transformar y crear a partir del material-para cualquier propósito, incluso para fines comerciales, siempre que cumpla las condiciones de Atribución. Usted debe dar crédito a la obra original de manera adecuada, proporcionar un enlace a la licencia, e indicar si se han realizado cambios. Puede hacerlo en cualquier forma razonable, pero no de forma tal que sugiera que tiene el apoyo del licenciante o lo recibe por el uso que hace de la obra.

$\underline{\text { Resumen de licencia }- \text { Texto completo de la licencia }}$ 